Accelerator Division

Alternating Gradient Synchrotron Department BROOKHAVEN NATIONAL LABORATORY

Associated Universities, Inc.

Upton, New York 11973

Accelerator Division

Technical Note

AGS/AD/Tech. Note No. 361

SELECTION OF THE OPTIMUM CHARGE STATE FROM AN EBIS

K. Prelec

June 4, 1992 


\title{
SELECTION OF THE OPTIMUM CHARGE STATE FROM AN EBIS
}

\author{
K. Prelec
}

A high charge state, heavy ion source, followed by an RFQ and a linac (preferably superconducting), has been considered as an alternative injector for the BNL Booster $(1,2)$ replacing tandem accelerators. Although there are presently three approaches in the design of such a source, namely an electron cyclotron resonance source (ECR), an electron beam ion source (EBIS), and a laser source, the most promising seems to be the EBIS. Recently some funds were received to start an EBIS project in the AGS Department; this note will address the question of one of the design parameters of the source: selection of the most abundant charge state in the ion beam extracted from the EBIS, that one day could serve as part of the Booster injector. Several EBIS parameters (electron beam current density, confinement time, electron beam energy) depend on the requested most abundant charge state.

One of the most important criteria for the selection of this charge state is the number of fully stripped ions injected per pulse: into the AGS and, further, into the RHIC. This number will depend, among others, on the following:

a) Parameters of the EBIS; an EBIS delivers an ion beam with a certain charge state distribution, where the most abundant charge state depends basically on the product of the electron beam current density and ion confinement time, while the total number of positive charges in the extracted beam will depend on the total electron space charge in the device. From this it follows that in an EBIS the number of ions in the selected (most abundant) charge state: will be, to the first order, inversely proportional to the charge state of the ion.

b). Injection energy into the Booster; this energy will depend on the design of the linac and is, at least at this moment, a free parameter which can in principle be selected to match the Booster requirements at injection (space charge limit, etc.). Therefore, we will assume: that the transfer efficiency, including capture, between the EBIS and the Booster does not depend on the selected charge: state of the ion at injection. However, if there is a dependency, this effect can be later on taken into account as a multiplying factor.

c) output energy of the Booster; for a fixed value of the Booster rigidity equal to: 17.5 . T-m one can calculate the corresponding values of the output ion energy as function of the charge state. Some data on the fraction of bare ions of $A u$ and $U$, after passing 
through a stripper and as function of the ion energy, are available from: experiments at LBL (Fig 1; private comm.). They show a fast rise above a few hundred $\mathrm{MeV} / \mathrm{amu}$, approaching a saturation above 500-600 MeV/amu. By using these results one can calculate the fraction of bare nuclei in the beam after passing through a stripper placed between the Booster and AGS, as function of the initial charge state (which is the most abundant charge state from the source). This will again be a function rising at first faster and then; above about 500-600 MeV/amu, more slowly.

There are, therefore, two important effects that will determine the number of fully stripped ions at injection into the AGS: on one hand the number will be inversely proportional to the value of the selected charge state from the source, but on the other it will be a rising function of the charge state as described above, under c). By dividing the values of the fraction of bare nuclei (after the stripper) with the value of the initial charge state we should obtain a measure for the number of fully stripped ions, as function of the initial charge state.

This estimate was done for two species, gold for the range of initial charge states between $32+$ and $43+$, and uranium for the range between $36+$ and $56+$; results are shown on Fig.2. In the past (Ref.1) we have considered initial charge states from the EBIS as high as $51+$ for gold and $64+$ for uranium. From the present results it follows that a charge state for gold as low as $36+$ to $40+$ would be quite sufficient, while for uranium this range would be $46+$ to 50+. This change certainly relaxes the design of the EBIS, especially the requirements for the product of the electron beam current density and ion confinement time.

There are, however, several effects so far neglected that may change the conclusions:

a) The charge state distribution of ions extracted from an EBIS may show effects of stripping to a closed shell or sub-shell. This has been observed with lighter elements when the stripping has reached inner shells, with the result that the abundance of such ions is higher than of those with incompletely filled shells. There are doubts that this effect would be very pronounced in very heavy ions; for partial stripping of the N-shell; also, there are no experimental data that could confirm it. If, however, in the mean time an appreciable effect of this nature is discovered, it would favor the selection of charge states $33+$ or $43+$ for gold and $46+$ or $56+$ for uranium.

b) There are indications that the available number of positive charges from an EBIS may slowly decrease with the confinement time. 


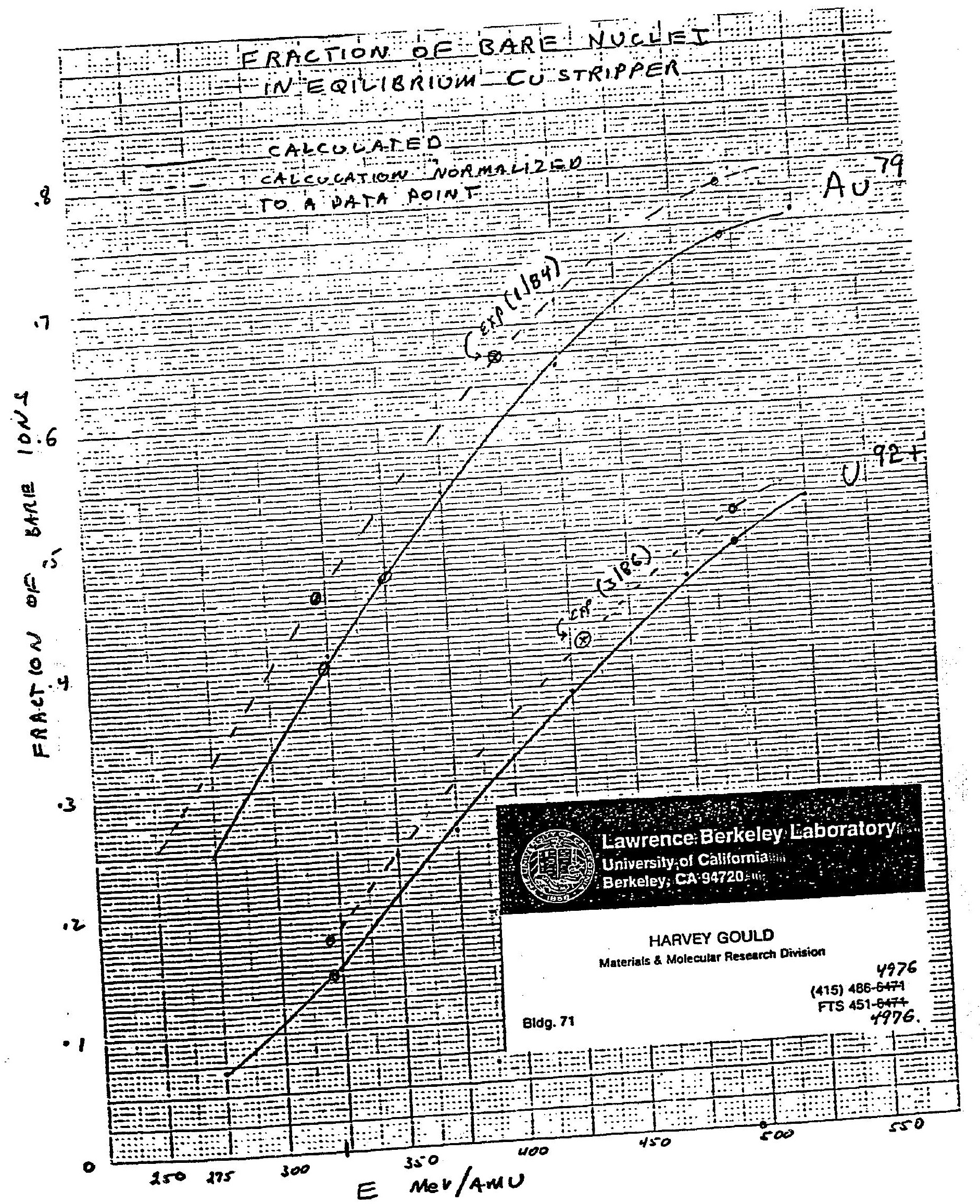


Such an effect would tend to shift the optima on Fig.2 toward lower values of the charge state. Combined with a), this would favor a charge state $33+$ for gold and $46+$ for uranium.

c) It will be necessary to study multi-pulse injection from the linac into the Booster (scheme applied at MIMAS-SATURNE) because this may be preferable than the design of a much larger and more powerful EBIS. However, with the design envisaged for the linac (a number of individually phased cavities) it should be possible to minimize the effect of the ion charge state on the parameters of the beam at injection into the Booster and the study should be done for a general case only.

d) Effects of the stripping foil on the phase space parameters of the fully stripped beam should be investigated, as function of the Booster output energy, i.e. of the initial charge state.

\section{References:}

1. K. Prelec, Synchrotron injectors based on high charge state ion sources, BNL-43944 (Feb.1990)

2. K. Prelec, M.J. Rhoades-Brown, P. Thieberger and H.E. Wegner, Comparative performance study of heavy-ion injector systems for high-energy synchrotrons, Nucl. Instr. Meth. in Phys. Res., A295 $(1990), 21-33$ 